\title{
Electrochemical determination of 3-nitrophenol with a reduced graphene oxide modified screen printed carbon electrode
}

\begin{abstract}
An electrochemical sensor based on electrochemically reduced graphene oxide (ERGO) functionalized cetyltrimethylammonium bromide (CTAB) deposited on screen printed carbon electrode (SPCE) was developed for the detection of 3-nitrophenol. The ERGO/CTAB was prepared via drop casting technique on the screen printed carbon electrode (SPCE). The drop casted composite was then subjected to cyclic voltammetry technique to produce ERGO/CTAB. The modified electrode exhibits high electrocatalytic activity and good selectivity towards the reduction of 3-nitrophenol due to its excellent electrical conductivity, strong adsorptive ability and large effective surface area of ERGO functionalized CTAB. The enhancement factor of the ERGO/CTAB modified SPCE towards 3-nitrophenol was calculated to be 5.16 times higher compared to bare SPCE. Under optimum experimental conditions, the linearity of the sensor towards 3-nitrophenol concentration was in the range of $0.5 \mu \mathrm{M}$ to $100 \mu \mathrm{M}$ with the detection limit of $0.04 \mu \mathrm{M}$. Furthermore, the ERGO/CTAB sensor showed good reproducibility and also demonstrated efficiency in the detection of 3nitrophenol in water samples.
\end{abstract}

Keyword: Electrochemically reduced; Environmental; Graphene oxide; Nitrophenol 